\title{
Are the "Physically Bound Systems" beyond the Cosmological Expansion Effect? (A provocative musing on the given theme)
}

\author{
Haik A. Harutyunian \\ Byurakan Astrophysical Observatory (BAO), Armenia \\ Email: hhayk@bao.sci.am
}

\begin{abstract}
Influence of the dark energy at small scales is considered. Interaction and energy exchange between ordinary matter and dark energy is proposed as a working hypothesis. Some observational facts are put into the base of this consideration: the large rate of lunar retreat and the acceleration of cosmic expansion which proves the energy exchange between ordinary matter and dark energy. If the possibility of the space and matter expansion at the scales under consideration is accepted one can show that the dark energy transformed into the object's potential energy is enough to generate cluster of galaxies over the Hubble time due to matter ejection mechanism.
\end{abstract}

Keywords. Cosmological expansion - Moon retreat - Dark energy

\section{Introduction}

All the energy forms we know about and we deal with are accepted, according to modern cosmological ideas, to be transformations of the original big bang energy. It concerns also the energy of various types of activity in cosmic objects. Tough this assertion is not loudly spoken out at least, in the form expressed here, the logics of the adopted cosmological theory based on the big bang hypothesis inevitably leads to such a conclusion. It is evident then that after the first release of energy which triggered the creation of the Universe only transformation of the total amount of the primary (kinetic) energy is going on.

On the other hand, by the discovery of the Universe accelerated expansion a new "actor" has been involved into the old cosmological game. This new source is represented by the dark energy making up at present about 70 percent of the Universe total massenergy. This form of the energy though not understood completely displays some physical manifestations making it detectable. And first of all its interaction with or its influence on the ordinary matter should be mentioned as the most essential for the latter one. Obviously, no any acceleration effect could be detected if there was no interaction between these two substances. So, one may assert then, that besides the big bang initial energy of explosion additional quantities of energy are somehow transmitted continuously to the ordinary matter over time. It is apparent that the observed acceleration is a result of the work persistently implemented by the dark energy upon the ordinary baryonic matter.

All the statements above are provable if the dark energy concept is accepted. However, the issue of the space scale where the dark energy effect is recognized remains unclear. For the large scales the effect of acceleration is beyond of any doubt (or less doubt). And on the contrary, the researchers are very skeptical about the possible existence of acceleration effects at smaller scales. Actually this skepticism is not well grounded, and 
most probably such an attitude is resulted from the dominant view on the cosmic objects' formation mechanism.

On the other hand, there are observational facts arguing in favor of Hubble flow at much smaller scales. Though the majority of researchers do not accept the concept that so called cosmological expansion might be observed at small distances this approach seems to be rather fruitful for understanding many observational facts inexplicable or poorly explainable in the frame of conventional theories. Moreover, it seems that the expansion named cosmological has its origin in micro dimensions.

\section{What we observe at the shorter scales?}

Hubble expansion discovered for the cosmological scales is an extremely tiny effect if considered for short scales and short time periods. Calculated for the unit length it amounts to $2.42410^{-18} \mathrm{sec}^{-1}$ or $7.6510^{-11} \mathrm{yr}^{-1}$ for the Hubble constant value of $75 \mathrm{~km} / \mathrm{sec}$ per Mpc. Therefore, it is natural that this extremely small effect has been revealed first only for cosmological scales. Within our Galaxy and especially in the Solar system this effect, if any, should be utterly weak for direct observations. It is easy to find that the average distance between San and Earth should increase on about $11.5 \mathrm{~m}$ per year if Hubble expansion takes place at these scales and if no any mass change of the Sun-Earth system is occurring. The increase of lunar orbit would be $2.92 \mathrm{~cm}$ per year for the same assumptions about the physical conditions.

The only experimentally reliable effect of expansion we deal within the solar system is the lunar retreat. Recent series of lunar laser ranging (LLR) gave for the rate of Moon's removal $3.82 \pm 0.07 \mathrm{~cm}$ per year (Dickey et al. 1994) for our epoch. Traditionally this effect is explained due to the tidal effects which transfer some portion of the Earth spin to the Moon. The Earth's diurnal deceleration rate is $1.8 \mathrm{~ms}$ per century if astronomical chronology of registered eclipses of last two thousand years is taken into account (Morrison \& Stephenson 2002).

It is not difficult to calculate that a removal speed depends on the deceleration rate according to the following linear relation (see, for example, Harutyunian 1995):

$$
\frac{\Delta a}{a}=0.406 \frac{\Delta T}{T}
$$

where $\mathrm{a}$ is the orbital radius of Moon and $\mathrm{T}$ is the diurnal period of the Earth. Then it is clear that owing to tidal effects the speed of the lunar retreat would not exceed the value of $3.26 \mathrm{~cm} \mathrm{yr}^{-1}$. Let us stress again that this value represents the upper limit for the lunar retreat provided by transfer of angular momentum from the Earth without of any losses, and it appears to be far below the observed one.

Besides this there are several estimates of lunar orbital changes derived on the base of paleotidal records. These data give essentially small value for lunar retreat and support the validity of the derived length of day (l.o.d.) of 21.9 hours at $620 \mathrm{Ma}$. Then the value of $2.16 \mathrm{~cm} /$ year since $\sim 620 M a$ is derived for the mean rate of lunar retreat Williams (1997). However, the fact that the lunar retreat rate derived for the past 0.62 Gy appears to be smaller compared with its present rate should obtain a self-consistent explanation. It is obvious that the Earth's angular momentum loss must decrease in the course of the Moon's removal. And therefore, the farther is the Moon from the Earth, the lesser should be its retreat rate. Obviously, the relation $\frac{\Delta T}{T}$ tends to the zero and consequently it should decrease over time. Then why we observe the monotonic increase of this value over time? Two possible answers might be considered - measurements are not correct or not an adequate mechanism of lunar retreat is used. 


\section{What happens if we include the universal expansion?}

Let us accept the observational data concerning the lunar retreat to be correct. This is reasonable owing to the unceasing improvement of measurement precession during last four-five decades which made all the relevant data very confident. Moreover, we hypothesize that the Hubble expansion is not the prerogative cosmological scales only but it has more universal behavior and takes place at all measurable scales. Then the Hubble law is rewritten in the form

$$
\frac{d r}{d t}=H r
$$

the exponential solution of which gives for short time scales

$$
r=r_{0}\left[1+H\left(t-t_{0}\right)\right]
$$

Calculation for the orbital radius of the Moon yields the value $2.92 \mathrm{~cm}$ for its annual increase which is below the observed one by $0.9 \mathrm{~cm}$. The situation seems to get even worse than that got for the purely tidal mechanism because now the Earth's expansion equal to $0.48 \mathrm{~mm}$ per year should be taken into account (compare it with forgotten $0.4-0.66 \mathrm{~mm}$ per year obtained by (Egyed 1956) using methods of palaeogeography). It results the Earth's diurnal rotation additional deceleration which can be easily found. The angular momentum of the Earth is given by the formula

$$
I_{g l o b}=0.83 \frac{4 \pi}{5} \frac{M R^{2}}{T}
$$

which gives $1.3 \mathrm{~ms}$ per century for the additional deceleration. However, notice that now there is reserved also some portion of the Earth angular momentum (corresponding to the deceleration rate equal to $1.8-1.3=0.5 \mathrm{~ms} \mathrm{cy}^{-1}$ ) left for the tidal effects again. It is easy to find that the upper limit for lunar retreat due to this portion amounts to $0.9 \mathrm{~cm}$ $\mathrm{yr}^{-1}$. Therefore, the joint effect of two mechanisms provides the speed of $3.82 \mathrm{~cm} \mathrm{yr}^{-1}$ for lunar retreat which fits the observational data with an unbelievable accuracy. Is this absolute accordance another amazing game of chance? That's worth thinking over.

\section{Dark energy transmission to the cosmic objects}

Since the end of last century a new "actor" has been involved into the old cosmological game, namely, the dark energy making up at present about 70 percent of the Universe total mass-energy. This form of the energy though not understood completely displays some physical manifestations making it detectable. And first of all its interaction with or its influence on the ordinary matter should be mentioned. Obviously, no any acceleration effect could be detected if there was no interaction between these two substances. So, one may assert then, that besides the big bang initial energy of explosion additional quantities of energy are somehow transformed continuously to the ordinary matter over time. It is apparent that the observed acceleration is a result of the work persistently implemented by the dark energy upon the ordinary baryonic matter.

Let us now consider a spherical cosmic object interacting with the dark energy through the expansion of the space. One can examine the potential energy change when such an object follows the cosmic expansion according the formula (3.2). The potential energy for a spherical object with given radius $R$ and density distribution $\rho(r)$ can be calculated easily to find

$$
U=-k_{U} G \frac{M^{2}}{R}
$$


where $M$ is the total mass of the object and the coefficient $k_{U}$ depends only on the mass distribution. The energy change due to the expansion effect if no other parameters change for the time interval $d t$ amounts to

$$
d U=k_{U} G \frac{M^{2} H d t}{R(1+H d t)} .
$$

On the other hand it is easy to see that a part of the relation (4.2) rewritten in the form

$$
v^{2}=\frac{2 G M}{R(1+H d t)}
$$

represents the square of the escape velocity from the surface of the expanded object. Therefore, one arrives at a conclusion that the energy accumulated owing to expansion is enough for giving an amount of kinetic energy to the mass

$$
d M=k_{U} M H d t
$$

for escaping from the maternal object.

According to current data, the masses of galaxy clusters are of about $10^{14}-10^{15}$ solar masses. It is easy to verify that a single protocluster with this much mass could eject an object with $10^{11}-10^{12}$ solar masses over $10^{7}$ years. Thus, this mechanism could provide the galaxy clusters' formation due to matter ejection for the Hubble time.

Analogous calculations could be done for other cosmic objects and systems. Another interesting problem arises if one tries to understand how could affect the dark energy atomic nuclei. Of course, for the conventional science this case sounds as a flat nonsense, even more than one we considered here. However, no evidence exists that there is no exchange between the dark energy and nuclear binding energy. And therefore the case should be considered carefully.

\section{References}

Dickey, J. O., Bender, P. L., Faller, J. E., Newhall, X. X., Ricklefs, R. L., et al. 1994, Science 265,482

Egyed, L. 1956, Nature 178, 534

Harutyunian, H. 1995, Astrophysics 38, 667

Morrison, L. V. \& Stephenson, F. R. 2002, in Rickman, H., ed., Highlights of Astronomy 12, 338

Williams, G. E. 1997, Geophysical Research Letters 24, 421 